\title{
COVID-19 in China: the role and activities of Internet-based healthcare platforms
}

\author{
Da He', Yichun Gu', Ying Shi', Miao Wang ${ }^{2}$, Zhexun Lou ${ }^{3}$, Chunlin Jin ${ }^{1, *}$ \\ ${ }^{1}$ Shanghai Health Development Research Center, Shanghai, China; \\ ${ }^{2}$ Shanghai Children's Hospital, Shanghai, China; \\ ${ }^{3}$ China Pharmaceutical University, Nanjing, China.
}

\begin{abstract}
In the fight against the COVID-19 epidemic, the Chinese Government has enhanced its use of Internetbased healthcare. A large number of online medical platforms designed for COVID-19 have emerged in China. These platforms can be categorized according to the entity operating them, mainly the government, hospitals, and companies. Online medical platforms run by public hospitals provide follow-up consultations for common ailments and frequent ailments based on the hospital's offline services. Online diagnosis and treatment platforms provided by companies cover most of the regions in China. In terms of offering pandemic-related services, corporate platforms provide at least 1,636,440 doctors for online care, 1.685 billion consultations, and 109 million remote consultations. In terms of regular medical care, those platforms provide at least 940,182 doctors for online care and 13.7 million remote consultations; more than 84,916 specialists have provided online care during this period. During the prevention and control of this epidemic, online diagnosis and treatment has filled the gap of family doctors in epidemic prevention and control, it has reduced the chance of cross-infection of patients with a mild infection, and it has overcome the geographical limitations of medical resources. However, online diagnosis and treatment still faces challenges in terms of resource allocation and industry supervision.
\end{abstract}

Keywords: COVID-19, SARS-CoV-2, China, Internet-based healthcare, online medical platforms

\section{Introduction}

As of March 31, 2020, the WHO reported a total of 750,890 confirmed cases of COVID-19 worldwide, of which 82,545 were diagnosed in China; 36,405 people died worldwide, of which 3,314 died in China (1); there are still 20,314 individuals who had close contact with SARS-CoV-2 who are still under medical observation in China (2).

Since the end of 2019 when pneumonia caused by SARS-CoV-2 spread in Wuhan, Hubei, the central government and the regional governments prioritized this issue, and the healthcare industry has stood at the forefront of the battle against the epidemic (3). In order to prevent the spread of the virus, the government and disease prevention and control departments have joined forces with multiple departments to raise citizens' awareness of self-protection from the disease. All provinces have implemented a primary public health emergency response, requiring people to stay at home as much as possible, travel less, cancel mass gatherings, and avoid crowds. Even during the Chinese Spring Festival, the country's most revered tradition, the government explicitly suggested canceling New Year's greetings (4). In the face of the panic brought about by this unknown disease, online medical platforms designed for COVID-19 have emerged, providing online care and information like updates on the epidemic, scientific articles and videos, dispelling rumors, clinic maps, online psychological assessments, tools to test for symptoms of pneumonia, instruction manuals, drug delivery services, free clinics for COVID-19, observation of at-home quarantine, psychological counseling and hot lines, and health insurance information (5-9).

In addition to pneumonia caused by coronavirus, many patients have chronic diseases, common ailments, seasonal diseases, or need tumor treatment, immunization, etc. Medical needs of these groups of patients may not be satisfied due to the fear of crossinfection in hospitals or travel restrictions. They may not receive timely treatment or medication for long-term treatment (10). In order to support Hubei, numerous teams of doctors and nurses from Respiratory Medicine, Infection Control, ICUs, ENT, Dentistry, and traditional Chinese medicine (TCM) departments across the country have set out to assist Wuhan, resulting in a shortage of medical resources in hospitals outside Hubei. Certain 
departments in some hospitals have suspended services (11). In addition, clinics in medical facilities around the country have overburdened with too many patients as the epidemic spread. Some hospitals have increased the proportion of online medical care (12). In this context, Internet-based healthcare has regained its significance.

\section{Concept behind and operation of Internet-based healthcare}

\section{Concept behind Internet-based healthcare}

Internet-based healthcare is the combination of the Internet and complete medical care. It involves seven main entities: the Internet, doctors, patients, hospitals, pharmaceutical companies, logistics companies, and insurance providers. It uses the Internet as a conduit and technical means to provide health and medical care including health education and medical information, electronic health records, assessment of disease risk, online disease consultation and diagnosis, remote consultation, remote rehabilitation, electronic prescribing, and drug delivery.

The core of the Internet is interconnected information. For patients, the Internet can solve the problem of insufficient communication between patients and doctors due to time limits, and it can fix the problem of having to go to the hospital in person for medical care in terms of appointments. For doctors, it can extend their available hours, increase the work experience of young doctors, increase their income, and highlight the value of their profession. For hospitals, operational efficiency can be improved, appointments and registrations can be made online, thereby improving patient satisfaction; for administrative departments, consulting or prescribing medicine online can provide a massive amount of medical data, which can facilitate disease management, drug development, hospital management, and medical insurance adjustment. The process of Internet-based healthcare is shown in Figure 1.

A description of the mode of operation of typical online diagnosis and treatment platforms in China

At present, online medical platforms can be categorized into government-based, hospital-based, and corporate platforms.

Online diagnosis and treatment platforms built by the government are mainly led by local governments. They commission Internet companies to develop, design, and/ or operate and maintain the platform. They also organize doctors at hospitals in the region to provide patients with free information, science education, and online consulting services. A typical representative of this type of platform is an online consultation platform in Beijing.

Online diagnosis and treatment platforms built by hospitals can be divided into two categories according to the extent of the part played by hospitals and Internet companies in the platforms. The first type is a platform launched and built by a hospital, which is actually an online extension of a hospital, as exemplified by the Online Hospital of Shanghai Children's Hospital (13). The second type is jointly launched by one or several hospitals and Internet companies. In this type, the Internet companies build third-party platforms and the hospitals arrange medical staff to provide online care on the platform and perform continuous diagnosis and treatment offline. The two parties reach an agreement on the rights and obligations in relation to the operation of the online hospital. Online hospitals including the
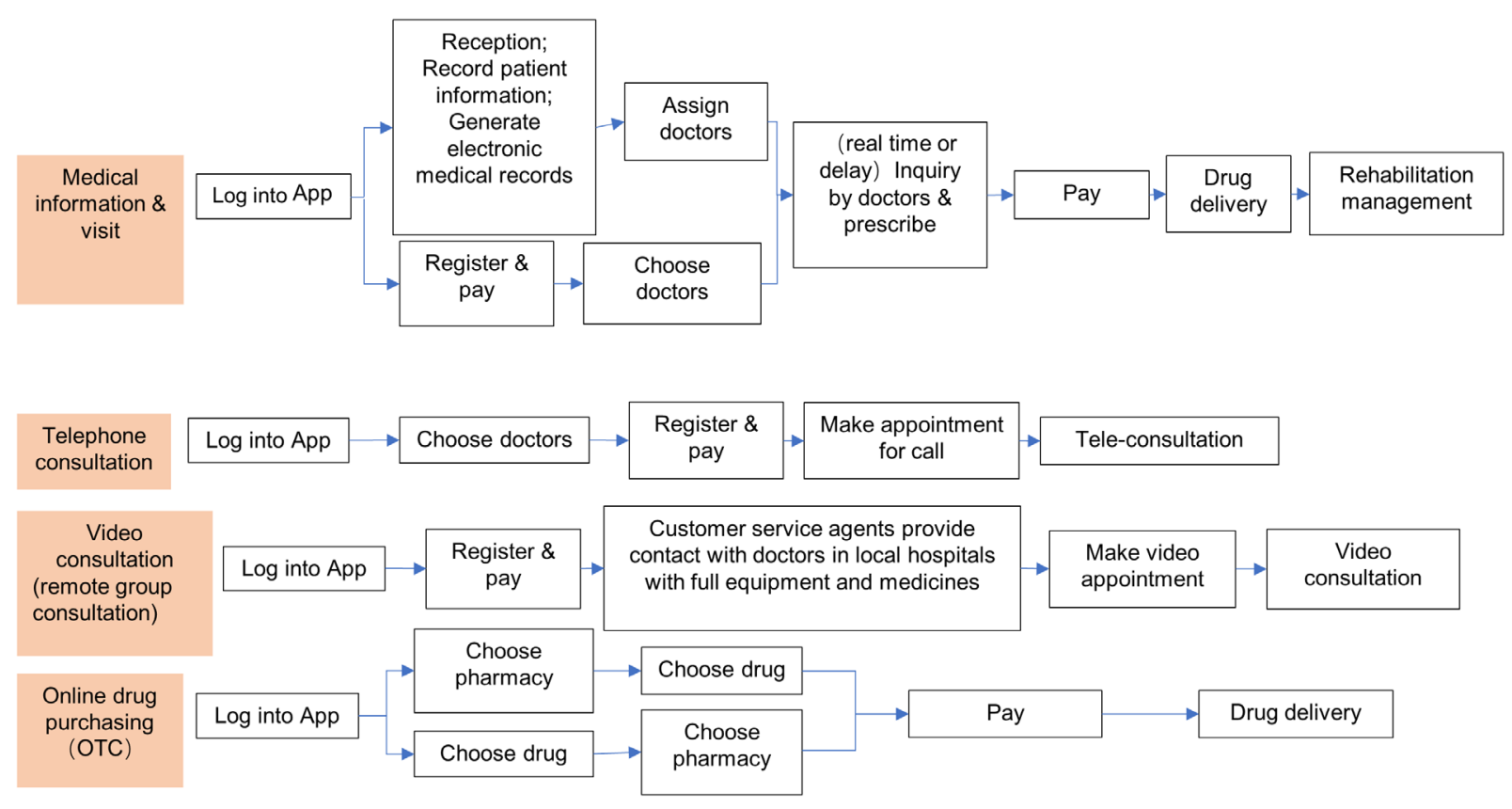

Figure 1. Flow chart for Internet-based healthcare. 
Ningxia Yinchuan Online Hospital, Shanghai Xuhui District Central Hospital Guanzhong Online Hospital, and Tianjin We Doctor Online Hospital exemplify this type of platform (14-17).

Online diagnosis and treatment platforms built by companies are often launched by Internet companies. This type of platform establishes or purchases a private hospital depending on its online medical platform. In a few cases, they also rely on public hospitals to assemble doctors in various places. Doctors provide services such as diagnosis and treatment by working with multiple sites on the platform. In principle, the doctor's work is regarded separately from his or her work at a facility. Wuzhen Online Hospital, Ali Health, Tencent Penguin Hospital, Good Doctor, Ping An Good Doctor, and Lilac Garden exemplify this type of platform (18-22).

\section{Services and effectiveness of Internet-based medical care during the COVID-19 outbreak}

When fighting the epidemic, the online diagnosis and treatment platforms run by the government and by hospitals provide care similar to that offline. Table 1 lists some of the services provided by some of the relatively large online diagnosis and treatment platforms operated by companies.

Treatment of COVID-19 has affected normal care at high-level hospitals (tertiary general hospitals and specialized hospitals) since these hospitals are usually designated to treat COVID-19 patients in most areas of China. Such an arrangement also leads to failure to meet routine medical needs for chronic diseases, common ailments, seasonal diseases, tumor treatment, immunization, etc. (23). Table 2 describes the activities the online diagnosis and treatment platforms run by companies in terms of diagnosis and treatment of conventional diseases.

Support and promotion of national policies related to online diagnosis and treatment in the prevention and control of the COVID-19 epidemic

China issued policies to use the Internet to combat the COVID-19 epidemic within one month of its outbreak. On February 7, 2020, the Government issued polices "to capitalize on the advantages of online medical care and to vigorously develop online diagnosis and treatment services, and especially online diagnosis and treatment consulting services for patients with a fever." All provincial health and administrative departments are directed to establish a uniform online medical care platform, to organize certified medical facilities in the province, and to organize doctors from Respiratory Medicine, Infection Diseases, Emergency Medicine, ICUs, Mental Health, and general departments to provide online consultation services for patients with a fever. According to these policies, certified medical facilities

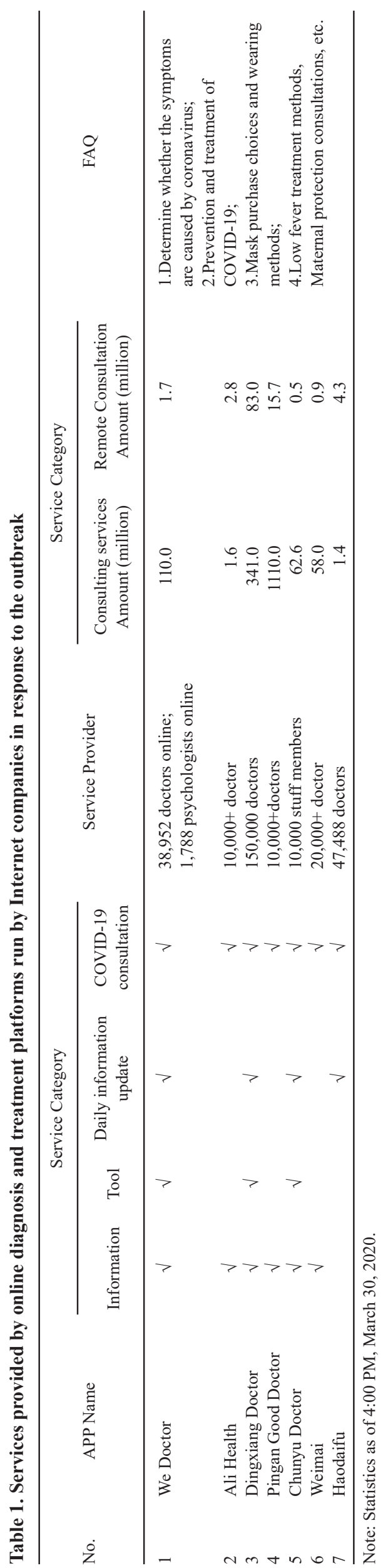




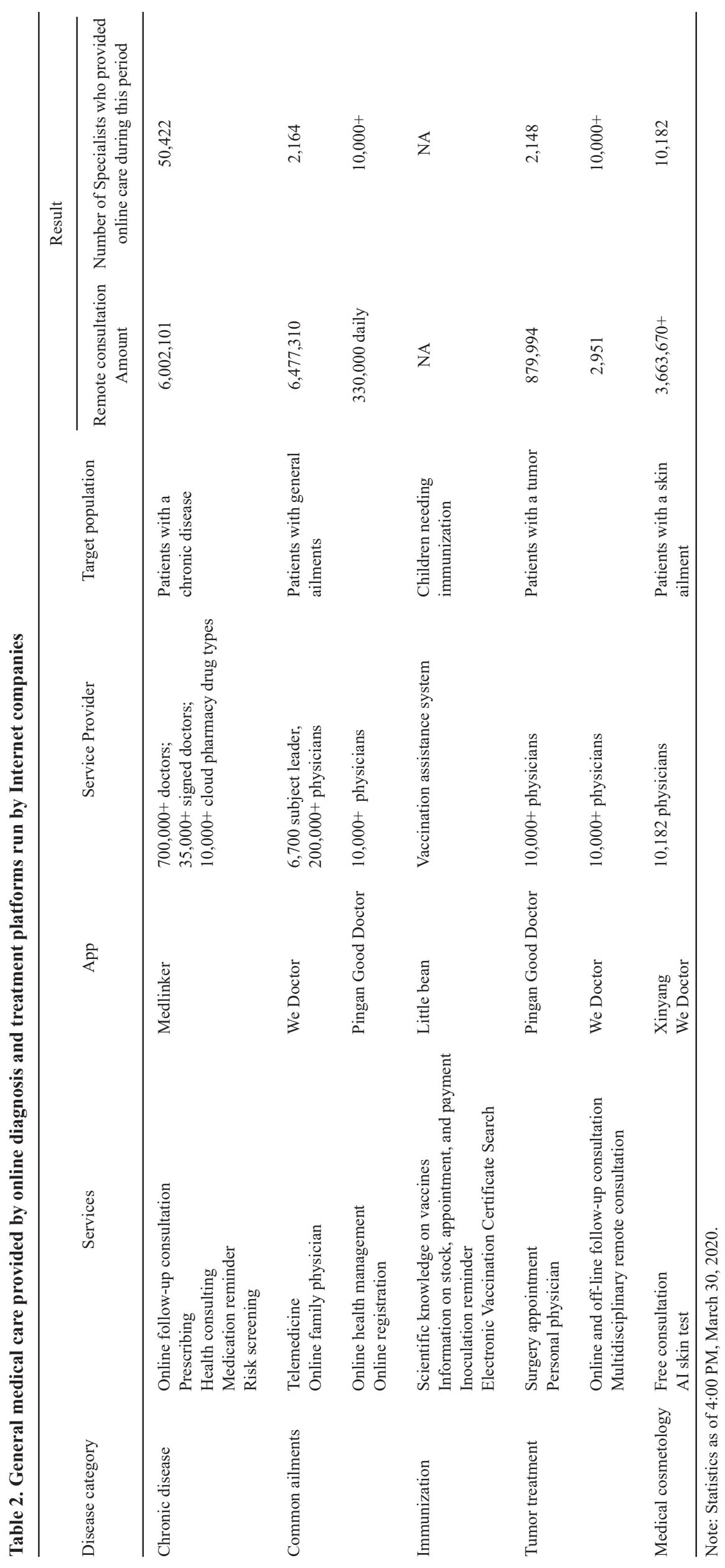

(92) 


\section{Table 3. China's policies on online diagnosis and treatment in response to the COVID-19 outbreak}

\begin{tabular}{|c|c|c|c|}
\hline No. & Release time & Policy & Department \\
\hline 1 & February 7, 2020 & $\begin{array}{l}\text { Notice on the Hard Work of Online Diagnosis and Consultation } \\
\text { Services to Prevent and Control Epidemics (General office of the } \\
\text { National Health Commission Doc No. [2020] No. 112) (24) }\end{array}$ & $\begin{array}{l}\text { National Health Commission of the People's } \\
\text { Republic of China }\end{array}$ \\
\hline 2 & February 21, 2020 & $\begin{array}{l}\text { Notice on National Tele-Consultation Services at the National } \\
\text { Telemedicine and Connected Health Center for Severe or Critical } \\
\text { Patients with COVID-19 (General office of the National Health } \\
\text { Commission Doc No. [2020] No. 153) (25) }\end{array}$ & $\begin{array}{l}\text { National Health Commission of the People's } \\
\text { Republic of China }\end{array}$ \\
\hline 3 & February 26, 2020 & $\begin{array}{l}\text { Notice on Launching Online Services to Further Enhance Epidemic } \\
\text { Prevention and Control in Hubei (Comprehensive Prevention and } \\
\text { Control Mechanism [2020] No. 85) (26) }\end{array}$ & $\begin{array}{l}\text { Leading Group of the CPC Central } \\
\text { Committee for COVID-19 Prevention and } \\
\text { Control }\end{array}$ \\
\hline 4 & February 28, 2020 & $\begin{array}{l}\text { Guiding Opinions on Promoting "Internet +" Medical Insurance } \\
\text { Services during the Prevention and Control of the COVID-19 } \\
\text { Outbreak (29) }\end{array}$ & $\begin{array}{l}\text { National Health Care Security Administration, } \\
\text { National Health Commission of the People's } \\
\text { Republic of China }\end{array}$ \\
\hline 5 & March 13, 2020 & $\begin{array}{l}\text { (Tentative) Notice on the Formulation of a Health Management Plan } \\
\text { for Newly Discharged Patients with COVID-19 (General office of } \\
\text { the National Health Commission Doc No. [2020] No. 225) (27) }\end{array}$ & $\begin{array}{l}\text { National Health Commission of the People's } \\
\text { Republic of China }\end{array}$ \\
\hline
\end{tabular}

need to fully utilize their in-house platform, online medical platforms, their website, social media platforms, and other platforms to fast-track consultations for patients with COVID-19 or a fever, to provide free online consultations for COVID-19, to provide guidance for home-based medical observation and health assessment, and to provide other services to guide patients to receive medical care in an orderly and precise manner (24).

On February 21, the Chinese Government clarified the fact that the National Telemedicine and Connected Health Center (China-Japan Friendship Hospital) would take charge of operating a national teleconsultation platform for patients with severe COVID-19. Provincial health and administrative departments are required to guide designated hospitals in their jurisdictions to provide remote consultations for patients with severe COVID-19 to aid in their recovery (25). On February 26 , the Government put forward specific requirements in terms of standardizing online diagnosis and treatment services and it made specific suggestions for national medical resources to support Wuhan, Hubei via the Internet (26). On March 13, the Government emphasized Internet-based health management of discharged patients with COVID-19 (27).

In terms of medical insurance, the emergency code "RA01.0", which represents COVID-19, was added to the ICD-11 codes (28). Designated medical facilities that have been approved to establish an online hospital or online diagnosis and treatment by the health administrative department can be reimbursed for the cost of "Internet +" follow-up consultations by insured patients for common ailments and chronic diseases (29). Table 3 shows the Chinese national policies related to Internet-based healthcare during the outbreak.

The state has put forward a series of policies and measures supporting and promoting online diagnosis and treatment to prevent and control the COVID-19 epidemic, thus providing new opportunities for the development of online diagnosis and treatment. However, a point worth mentioning is that online diagnosis and treatment still faces obstacles such as a lack of medical and IT personnel (30), failure to connect patient information (31), unnecessary repetition and omission due to multi-sector supervision (32), regulatory obstacles such as whether online diagnosis and treatment should have the same standards as those of public hospitals (33), as well as challenges in terms of the security of patient information and medical ethics $(34,35)$.

\section{Conclusion}

During the prevention and control of this epidemic, online diagnosis and treatment has played three vital roles. First, it fills the gap of family doctors in epidemic prevention and control, including giving local residents timely medical consultations, performing triage, and providing emotional comfort and psychological counseling. Second, it reduces the chance of crossinfection of patients with a mild infection, that is, it physical isolates patients through remote diagnosis of disease. Third, it overcomes the limitation of medical resources in terms of space; tens of thousands of doctors have participated in epidemic prevention and control at different times and in different places (36). During the epidemic, some of the medical insurance payment problems that have long troubled the development of online diagnosis and treatment have been partially solved, but there are still many problems in terms of technology, supervision, and insurance that need to be fixed systematically. 


\section{References}

1. World Health Organization. Coronavirus disease 2019 (COVID-19) Situation Report-71. https://www.who.int/docs/ default-source/coronaviruse/situation-reports/20200331sitrep-71-covid-19.pdf?sfursn=4360e92b_8 (accessed March 31, 2020).

2. National Health Commission of the People's Republic of China. Update on the covid-19 outbreak as of 24:00 on 31 March. http://www.nhc.gov.cn/xcs/yqtb/202004/28668f9 87f3a4e58b1a2a75db60d8cf2.shtml (accessed March 31, 2020). (in Chinese)

3. National Health Emergency Office of the People's Republic of China. The National Health Commission and relevant departments endeavor to prevent and control the outbreak of COVID-19. http://www.nhc.gov.cn/xcs/ fkdt/202001/d9570f3a52614113ae0093df51509684.shtml (accessed January 21, 2020). (in Chinese)

4. Headquarters of SARS-CoV-2 Prevention and Control in Hubei Province. A Letter to the People of the Province. http://www.hubei.gov.cn/zhuanti/2020/gzxxgzbd/ $z x t b / 202001 / t 20200123$ 2014575.shtml (accessed January 23, 2020). (in Chinese)

5. Feng W, Zhang L, Li J, et al. Analysis of online health consultations for COVID-19. Journal of Peking University (Medical Edition), 2020; 1-14. (in Chinese)

6. Jiang Q. COVID-19 spurs the IT industry. China Internet Weekly, 2020; 70-71. (in Chinese)

7. Gao Y, Xu T, Jin Z, et al. Practice and discussion of outpatient pharmacy services based on a model of online medicine during the COVID-19 outbreak. Chinese Journal of Hospital Pharmacy, 2020; 40: 1-10. (in Chinese)

8. Shan N. Development and future of Internet-based health care amidst the COVID-19 epidemic. China Insurance. 2020; 2:40-42. (in Chinese)

9. Liu K. Ali health, Pingan Good Doctors, and We Doctor fight against COVID-19. Big Data Era. 2020; 60-65. (in Chinese)

10. $\mathrm{Yu}$ Q. Recommendations for remote consultations for patients with hypertension during epidemic prevention and control. China Rural Medicine. 2020; 27:31-33.

11. Yu Z. Can the status of the outbreak be regarded as "force majeure" for lifting of the quarantine? Chinese Health News. 2020-02-27 (006). (in Chinese)

12. Xin Q. Demonstration of online medicine. China Discipline Inspection and Supervision Daily, 2020-02-24 (004). (in Chinese)

13. Zhang L. Shanghai Children's Hospital creates an "Internet +" model for children's medical care. China Health, 2018; 105. (in Chinese)

14. Ningxia Daily. Yinchuan, Ningxia builds a top online hospital for industrial clusters. http://nx.people.com.cn/ n2/2018/0809/c192482-31913267.html (accessed February 22, 2020). (in Chinese)

15. Sina Financial Headlines. The Central Public Hospital of Xuhui District, Shanghai becomes the first public hospital in Shanghai to obtain an Online Hospital License. https:// cj.sina.com.cn/articles/view/1704103183/65928d0f02001ko ra? from=finance (accessed February 27, 2020). (in Chinese)

16. We Doctor website. https://tblife.guahao.com/ (accessed March 21, 2020). (in Chinese)

17. Wang Y, Zhang W, Wang W. An exploration of "Internet + Medical Health" in Yinchuan. Chinese Journal of Hospital Administration, 2019, 35: 623-626. (in Chinese)

18. Wuzhen Online Hospital website. https://wu.guahao.com/ (accessed March 21, 2020) (in Chinese)

19. Ali Health website. https://www.alihealth.cn/ (accessed March 21, 2020) (in Chinese)

20. Haodaifu website. https://www.haodf.com/ (accessed March 21, 2020). (in Chinese)

21. Pingan Good Doctor website. https://www.jk.cn/(accessed March 21, 2020). (in Chinese)

22. Deng Y. Examining a model of operating a Webconnected hospital and an exploration of legal issues. Chinese Health Quality Management. 2019; 4:113-116. (in Chinese)

23. Guo Y. Difficulties restoring order to medical care and countermeasures. http://www.yanghu123.com/ Article/2020/03/13/76265.html (accessed March 13, 2020). (in Chinese)

24. National Health Commission of the People's Republic of China. Notice on the Hard Work of Online Diagnosis and Consultation Services to Prevent and Control Epidemics. http://www.nhc.gov.cn/yzygj/s7653p/202002/ec5e34581 $4 e 744398$ c2adef17b657fb8.shtml (accessed February 27, 2020). (in Chinese)

25. National Health Commission of the People's Republic of China. Notice on National Tele-Consultation Services at the National Telemedicine and Connected Health Center for Severe or Critical Patients with COVID-19. http:// www.nhc.gov.cn/yzygj/s7658/202002/69b24672365043e ebc379c8bab30c90d.shtml (accessed February 21, 2020). (in Chinese)

26. Leading Group of the CPC Central Committee for COVID-19 Prevention and Control. Notice on Launching Online Services to Further Enhance Epidemic Prevention and Control in Hubei. http://www.nhc.gov.cn/guihuaxxs/ gongwen12/202002/91b668d87cd1443bbbalee9c706be1 4b.shtml (accessed February 26, 2020). (in Chinese)

27. National Health Commission of the People's Republic of China. (Tentative) Notice on the Formulation of a Health Management Plan for Newly Discharged Patients with COVID-19. http://www.nhc.gov.cn/yzygj/s7653pd/20200 3/056b2ce9e13142e6a70ec08ef970f1e8.shtml (accessed March 13, 2020). (in Chinese)

28. National Health Care Security Administration, National Health Commission of the People's Republic of China. Notice on the Issuance of ICD Codes related to SARSCoV-2 Infection. http://www.nhsa.gov.cn/art/2020/2/14/ art_37_2578.html (accessed February 14, 2020). (in Chinese)

29. National Health Care Security Administration, National Health Commission of the People's Republic of China. Guiding Opinions on Promoting " Internet + " Medical Insurance Services during the Prevention and Control of the SARS-CoV-2 Outbreak. http://www.nhsa.gov.cn/ art/2020/3/2/art_37_2750.html?from=timeline (accessed February 28, 2020). (in Chinese)

30. Meng S, Zhang W. Uses and challenges of creating digital medical information. Modern Information Technology. 2019; 3:101-102. (in Chinese)

31. Shi D. New challenges of "Internet + health". Business Culture. 2020; 78-83. (in Chinese)

32. Wang Y, Zhu Y. Challenges of and solutions to medical information security in the context of the Internet. Information and Computers (Theory Edition). 2019; 31:218-219. (in Chinese)

33. Wang M, Yu G. Impact and challenges of "Internet +" on Medical Care. Shanghai Pharmaceutical Corporation. 2017; 38:3-5. (in Chinese) 
34. China Population Daily. Discussion on the Ethical Issues of "Internet + Health". China Population Daily. 2019-1114(003). (in Chinese)

35. Zhao H, Luo J, Wang Z. Information security challenges in the Internet healthcare service model. China Digital Medicine. 2019; 14:92-93. (in Chinese)

36. Institute of Economics, Chinese Academy of Social Sciences. Fengmei Zhu: Internet-based medical care: The "second battlefield" for epidemic prevention and control. http://ie.cssn.cn/scholars/opinions_essays_ interviews/202002/t20200207_5086166.html (accessed February 27, 2020). (in Chinese)
$-$

Received March 16, 2020; Revised April 2, 2020; Accepted April 7, 2020.

Released online in J-STAGE as advance publication April 12, 2020 .

${ }^{\S}$ These authors contributed equally to this work.

*Address correspondence to:

Chunlin Jin, Shanghai Health Development Research Center, 181 Xinbei Road, Shanghai, China 201199.

E-mail: jinchunlin@shdrc.org 